\title{
Cloning of dapD, aroD and asd of Leptospira interrogans serovar icterohaemorrhagiae, and nucleotide sequence of the asd gene
}

\author{
CÉline Baril, Catherine Richaud, Edith Fournié, Guy Baranton and \\ ISABELLE SAINT GIRONS*
}

Unité de Bactériologie Moléculaire et Médicale, Institut Pasteur, 75724 Paris cedex 15, France

(Received 9 August 1991; accepted 11 October 1991)

\begin{abstract}
Metabolites such as diaminopimelate and some aromatic derivatives, not synthesized in mammalian cells, are essential for growth of bacteria. As a first step towards the design of a new human live vaccine that uses attenuated strains of Leptospira interrogans, the asd, aroD and dapD genes, encoding aspartate $\beta$-semialdehyde dehydrogenase, 3-dehydroquinase and tetrahydrodipicolinate $N$-succinyltransferase, respectively, were cloned by complementation of Escherichia coli mutants. The complete nucleotide sequence of the asd gene was determined and found to contain an open reading frame capable of encoding a protein of 349 amino acids with a calculated $M_{r}$ of 38007. Comparison of this deduced $L$. interrogans aspartate $\beta$-semialdehyde dehydrogenase amino acid sequence with those of the same enzyme from Saccharomyces cerevisiae and Corynebacterium glutamicum revealed $46 \%$ and $36 \%$ identity, respectively. By contrast, the identity between the $L$. interrogans enzyme and the Streptococcus mutans or $E$. coli enzymes was less than $31 \%$. Highly conserved sequences within aspartate semialdehyde dehydrogenase from the five organisms were observed at the amino and carboxyl termini, and around the cysteine of the active site.
\end{abstract}

\section{Introduction}

Leptospirosis, the disease resulting from infection with Leptospira interrogans, is one of the most widespread of the zoonoses. The major reservoirs for the pathogenic leptospires are rodents and domestic animals. The human vaccine, used in France, is composed of bacteria ( $L$. interrogans serovar icterohaemorrhagiae strain Verdun) that have been killed by formalin. An alternative would be to use a live vaccine based on attenuated organisms that are auxotrophic for metabolites not available in mammalian cells, such as aromatic amino acids or diaminopimelic acid (DAP). The genus Leptospira is an exception among the spirochaetes since DAP is found in its peptidoglycan while ornithine is found in the genera Spirochaeta, Borrelia and Treponema (Johnson, 1977). These flexible, helically shaped bacteria are very heterogeneous in regard to other characteristics.

* Author for correspondence. Tel. (1) 45688366 ; fax (1) 40560125 .

Abbreviation: DAP, diaminopimelate.

The nucleotide sequence data reported in this paper have been submitted to GenBank and have been assigned the accession number M77500.
Leptospires are aerobic and have simple nutritional requirements. The only necessary organic compounds are vitamins $B_{12}$ and $B_{1}$ and long-chain fatty acids, which serve as the obligate carbon and energy sources (Johnson, 1977).

The biosynthetic pathways for amino acids known for bacteria are used in leptospires except for isoleucine, which is not exclusively derived from threonine (Charon et al., 1974). Studies on the organization and regulation of the amino acid biosynthetic genes in Leptospira are still in their infancy. Three biosynthetic genes ( $\arg E$, $\operatorname{trp} G$ and $\operatorname{trp} E$ ) from the non-pathogenic species $L$. biflexa were cloned by complementation of the corresponding Escherichia coli mutants and then sequenced (Zuerner \& Charon, 1988; Yelton \& Charon, 1984; Yelton \& Cohen, 1986). The proA, proB and leuB genes from the pathogenic species $L$. interrogans were cloned by Richaud et al., 1990; the organization of the leu genes seems to differ from that in $E$. coli. The trpE gene from Spirochaeta aurantia, which also belongs to the order Spirochetales, has also been cloned (Brahamsha \& Greenberg, 1987).

Our strategy to obtain mutations in pathways leading to the biosynthesis of DAP and aromatic compounds in $L$. interrogans consists of cloning the biosynthetic genes 
by complementation of $E$. coli mutations, inactivating the cloned genes and then introducing the mutated genes back into $L$. interrogans, replacing the wild-type.

Here we report the cloning of the aroD, asd and dapD genes from $L$. interrogans serovar icterohaemorrhagiae strain Verdun. The nucleotide sequence of the asd gene specifying aspartate $\beta$-semialdehyde dehydrogenase (EC 1.2.1.11), an enzyme common to the biosynthetic pathways of threonine, methionine and DAP, is reported.

\section{Methods}

Bacterial strains, plasmids and media. L. interrogans serovar icterohaemorrhagiae strain Verdun (National Reference Centre, France) was grown in EMJH medium (Johnson, 1977). E. coli was grown in Luria Broth in liquid medium or $0.75 \%$ agar (Sambrook et al., 1989) supplemented with $50 \mu \mathrm{g}$ DAP ml-1 $50 \mu \mathrm{g}$ ampicillin ml $\mathrm{ml}^{-1}$ or $25 \mu \mathrm{g}$ chloramphenicol $\mathrm{ml}^{-1}$. Minimal medium 63 (Sambrook et al., 1989) supplemented with $0.2 \%$ glucose, $50 \mu \mathrm{g}$ ampicillin $\mathrm{ml}^{-1}$ or $25 \mu \mathrm{g}$ chloramphenicol $\mathrm{ml}^{-1}$ and $1 \mu \mathrm{g}$ thiamin $\mathrm{ml}^{-1}$ was used to characterize $E$. coli transformants. Supplements for growth of $E$. coli aro $D$ mutants were $40 \mu \mathrm{g} \mathrm{ml}^{-1}$ phenylalanine, tryptophan and tyrosine, $10 \mu \mathrm{g} \mathrm{ml}^{-1}$ $p$-aminobenzoic acid and dihydroxybenzoic acid. Other amino acids such as arginine or threonine were added at $40 \mu \mathrm{g} \mathrm{ml}^{-1}$. The cloned $E$. coli dap $D$ gene was inactivated by insertion of a chloramphenicol cassette from pSKS114 (Shapira et al., 1983) into its unique PstI site (Richaud et al., 1984). The $E$. coli asd gene was inactivated by insertion of a kanamycin cassette from pUC4K (Pharmacia) into the unique NruI site of the gene (Haziza et al., 1982). After transformation of the recBC sbcB E. coli strain JC7623 (Winans et al., 1985) by linearized plasmids carrying the inactivated genes, cells carrying the gene replacement were selected by their ability to grow in the presence of the antibiotic and DAP. The asd:: $\mathrm{Km}$ or dapD::Cm markers were then transferred to $E$. coli strain GT869 (Parsot, 1986) by Pl vir transduction to give GT2000 (asd) and GT2003 (dapD), respectively. E. coli G6MD3 is ( $\triangle a s d$ glpR glyD malA) his (M. Schwartz, Institut Pasteur, Paris, France).

Cloning of L. interrogans genes by complementation of E. coli mutants. Restriction enzyme digests, ligations, agarose gel electrophoresis, transformation, electroporation and small-scale plasmid isolations were by standard procedures (Sambrook et al., 1989).

Two different colony banks of, respectively, 8000 and 11000 individual $\mathrm{Ap}^{\mathrm{r}}$ transformants in $E$. coli strain GT869 were obtained from $L$. interrogans serovar icterohaemorrhagiae in the $E$. coli multicopy vector pUC13 as described previously (Richaud et al., 1990). In order to clone biosynthetic genes of the DAP and aromatic pathways from $L$. interrogans, complementation of the corresponding $E$. coli mutants was sought. A sample $(1 \mu \mathrm{g})$ of total plasmid DNA isolated from either clone bank was used to transform the $E$. coli $\operatorname{dap} D$, aroD and asd mutants.

Sequencing strategy for the asd gene. The $0.7 \mathrm{~kb}$ EcoRI fragment from pAIL 8 and the $2.7 \mathrm{~kb}$ EcoRI fragment from pAIL4 (see Fig. 1c) were cloned in both orientations into EcoRI-digested pTZ18R. A sequential series of overlapping deletions of the asd gene were obtained using an IBI Cyclone kit as described by Sambrook et al. (1989). The overlap of the internal EcoRI site was confirmed by sequencing the first 200 nucleotides of the $3 \mathrm{~kb} \mathrm{BamHI-HindIII} \mathrm{fragment} \mathrm{originating} \mathrm{from}$ pAIL4. The nucleotide sequence from the whole PvuI-SmaI insert from pAIL8 was determined by the dideoxy chain-termination method (Sanger et al., 1977) using a BRL sequencing kit. The sequencing vector
pTZ18R and helper phage M13K07 were purchased from Pharmacia. Acrylamide was purchased from $\mathrm{BDH}$, urea from Schwartz, and $\left[\alpha^{-35}\right.$ S $]$ dATP (14.8 TBq mmol-1) from Amersham. All other chemicals were analytical grade or purer.

\section{Results and Discussion}

Cloning of $L$. interrogans genes able to complement E. coli aroD, dapD and asd mutants

Complementation of $E$. coli mutants was used to isolate molecular clones of $L$. interrogans genes encoding enzymes of the DAP and aromatic pathways.

With the aroD E. coli strain GMS343 (Novel \& Novel, 1973) as recipient, 10 out of $40000 \mathrm{Ap}^{r}$ transformants were $\mathrm{Aro}^{+}$(able to grow without aromatic acid supplements). Analysis of the plasmid DNA (pADL1) of five of these clones by agarose gel electrophoresis after Bam HI digestion (Fig. $1 a$ ) indicated the same orientation of the $9.5 \mathrm{~kb}$ BamHI insert. Further subcloning and gene inactivation experiments allowed us to locate the aroD gene more precisely (see Fig. 1a). Other E. coli aroD mutants (AB1360 and AB2848) (Pittard \& Wallace, 1966) are complemented by pADLl, suggesting that the cloned gene is most likely the structural gene of the 3-dehydroquinase, the third step of the aromatic pathway.

With the dapD E. coli strain GT2003 as recipient, 17 out of $17000 \mathrm{Ap}^{r}$ transformants were $\mathrm{Dap}^{+}$(able to grow on LB medium without DAP). The plasmid DNA of nine of these clones was analysed by agarose gel electrophoresis after BamHI digestion. Six contained pDDLl (Fig. $1 b$ ) and the other three contained the same $9 \mathrm{~kb} \mathrm{BamHI}$ insert in the other orientation (pDDL2). Subcloning of pDDL1, first by Pst I digestion and religation to yield pDDL11 (Fig. 1b), then by BglII digestion of pDDL11 and religation, gave pDDL12, which was also able to complement the dapD mutation in GT2003. The plasmid pDDL12 also complemented another dapD mutant, RDD32, which carries a Mucts insertion in the $E$. coli dapD gene (Richaud et al., 1984). The $L$. interrogans DNA in pDDL12 is most likely the structural gene of tetrahydrodipicolinate $N$-succinyltransferase.

With the asd E. coli strain GT2000 as recipient, 5 out of $10000 \mathrm{Ap}^{r}$ transformants were able to grow on LB not supplemented with DAP. Each of these five carried a plasmid (pAlLl, see Fig. 1c) with a $19 \mathrm{~kb}$ insert that contained an internal BamHI site. Efficient complementation of an $E$. coli strain deleted for asd (G6MD3) by pAILl confirmed the presence of the asd structural gene on this plasmid. The asd complementing activity was still carried by a $1.8 \mathrm{~kb} S m a \mathrm{I}-P v u \mathrm{I}$ insert (plasmid pAIL8, see Fig. $1 c$ ). A $\mathrm{Cm}^{\mathrm{r}}$ cassette inserted at the internal BamHI site inactivated the gene. 
(a)

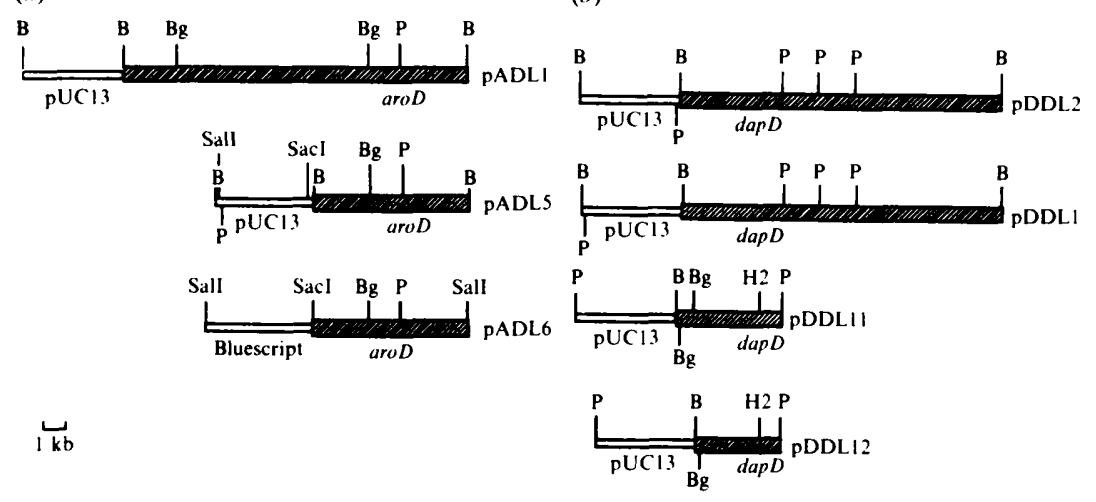

(c)

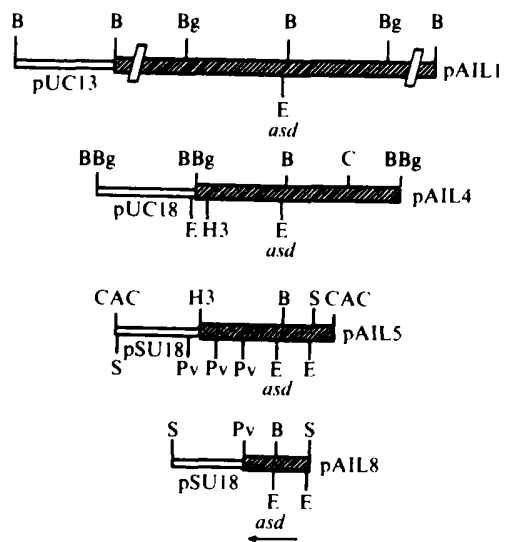

Fig. 1. Restriction maps of $L$. interrogans genes carried by plasmids. (a) Subclone analysis of aroD plasmids. pADLl contains a $9.5 \mathrm{~kb}$ $B a m H I$ insert in pUC13. pADL5 was obtained by deletion of the internal BglII fragment of pADL1, reducing the insert size to $4.8 \mathrm{~kb}$. The insert carried by pADL5 was ligated in the vector Bluescript (Stratagene) via SacI and SalI bordering sites to give pADL6. Inactivation of the aroD gene was performed by inserting a $\mathrm{Cm}^{r}$ cassette from $\mathrm{pSKS} 114$ into the unique PstI site of pADL6. (b) Subclone analysis of dapD plasmids. pDDL1 and pDDL2 correspond to both orientations of the $8.8 \mathrm{~kb} \mathrm{BamHI}$ insert in pUC13. Total digestion of pDDL1 with Pstl and self ligation gave rise to pDDL11; pDDL12 was obtained from pDDL11 by deletion of the internal Bg/II fragment. Inactivation of the dapD gene was performed by insertion of a $\mathrm{Cm}^{r}$ cassette from pSKSI14 into the unique HincII site of pDDL12. (c) Subclone analysis of asd plasmids. The plasmid pAILl contains a BamHI-BamHI insert of $19 \mathrm{~kb}$ (with a supplementary BamHI site) in pUC13. A $5.8 \mathrm{~kb} \mathrm{BglII-BglII}$ fragment from pAIL1 was cloned into the BamHI site of pUC18, giving rise to pAIL4. A $3.9 \mathrm{~kb}$ ClaI-HindIII fragment from pAIL4 was cloned between the AccI and HindIII sites of pSU18, giving rise to pAIL5. pAIL8 was obtained by two internal deletions ( $\mathrm{SmaI}$ and $P v u \mathrm{I}$ ) reducing the insert into the deleted pSU 18 to $1.7 \mathrm{~kb}$. B, BamHI; P,Pst $;$ Bg, BglII; H2, HincII; H3, HindIII; SalI SalI, SacI, SacI; E, EcoRI; BBg, BamHI/BglII; CAC, ClaI/AccI; S, SmaI. pSU18 encodes chloramphenicol resistance (Martinez et al., 1988); pUC13, pUC18 and Bluescript encode ampicillin resistance.

Hybridization analysis according to Southern (1975) was used to demonstrate that the DNA inserts of the $\mathrm{Asd}^{+}, \mathrm{Dap}^{+}$and $\mathrm{Aro}^{+}$recombinant plasmids originated from $L$. interrogans. The sizes of the Bam $\mathrm{HI}$ fragments which hybridized with $L$. interrogans chromosomal DNA were the same as those originating from the inserts (data not shown). The results thus indicate that no rearrangement had occurred in the $\operatorname{dap} D$, aroD or asd cloning experiments. Cloning of three $L$. interrogans genes involved in amino acid biosynthesis was thus possible by complementation of $E$. coli mutants. Characterization of one of them, the asd gene, was pursued further.

\section{Nucleotide sequence of the L. interrogans asd gene and flanking regions}

The sequence of the $1.8 \mathrm{~kb} S m a \mathrm{I}-P v u \mathrm{I}$ fragment from pAIL8 which complemented an asd E. coli mutant was determined on both strands and is presented in Fig. 2. Two large open reading frames (ORFs) were identified on one DNA strand. The first, ORF1, is located between nucleotides 1 and 392. The second, ORF2, later identified as asd, extends from nucleotide 393 to nucleotide 1493.

Codon usage for $L$. interrogans ORF1 and ORF2 is quite similar to that for the $\operatorname{trp} E$ and $\operatorname{trp} G$ genes from the saprophytic Leptospira biflexa (Yelton \& Peng, 1989) and for the sphingomyelinase gene from the pathogenic $L$. interrogans (Segers et al., 1990). The codon usage of $L$. interrogans is highly influenced by the low $\mathrm{G}+\mathrm{C}$ content $(34-39 \mathrm{~mol} \%)$ of its genome. $A$ or $T$ is the preferred nucleotide in the wobble position, except for Thr and Arg, where G is preferred, and Ala, where all four nucleotides are equally present.

ORF 1 is obviously incomplete since it does not contain an ATG or a GTG start codon. Comparison of the translated product of the 392-nucleotide ORF1 with the contents of the EMBL data bank did not show any significant homology with other known proteins. However, ORF 1 could be the $3^{\prime}$ end of a gene translated in the same direction as ORF2 and could form a potential operon with ORF2. The promoter of the operon would not be present on the SmaI-PvuI insert from pAIL8.

Comparison of Asd from L. interrogans, Corynebacterium glutamicum, Saccharomyces cerevisiae, Streptococcus mutans and E. coli

Comparison of the translated product of ORF2 with the contents of the EMBL data bank showed significant 


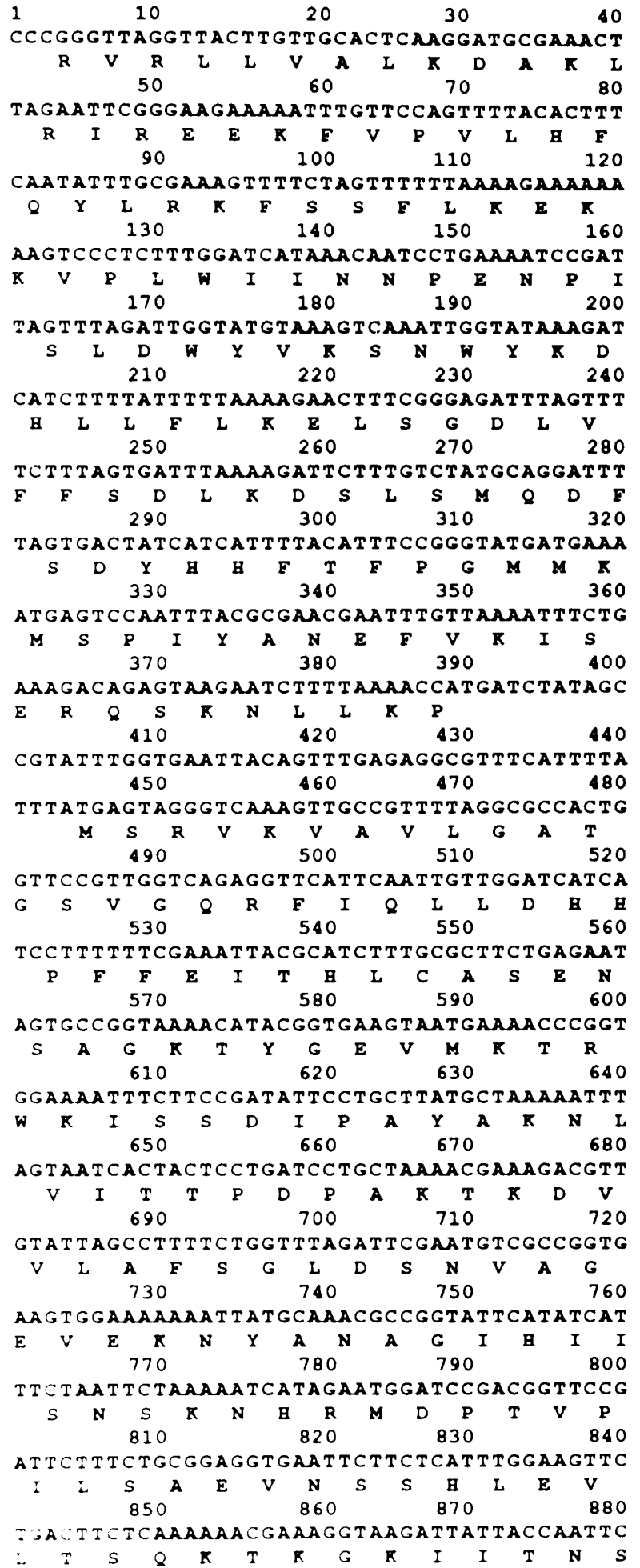

$\begin{array}{llll}890 & 900 & 910 & 920\end{array}$ GAACTGTACGATCATGGGTGTTACAATTTCTCTCAAACCT $\begin{array}{lllllllllllll}\mathbf{N} & C & \mathbf{T} & \mathbf{I} & \mathbf{M} & \mathbf{G} & \mathbf{V} & \mathbf{T} & \mathbf{I} & \mathbf{S} & \mathbf{L} & \mathbf{K} & \mathbf{P}\end{array}$ $930 \quad 940 \quad 950 \quad 960$ CTTTTGGATCGTTTTGGAATGAGTCCGTTATGCTCTTTT

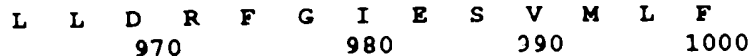
CTATGCAGGCGATCAGCGGCGCTGGTTATCCTGGAGTTCC $\begin{array}{llllllllllllll}S & M & Q & A & I & S & G & A & G & Y & P & G & V & P\end{array}$ $\begin{array}{llll}1010 & 1020 & 1030 & 1040\end{array}$ AACGATGGATATTTTAGGAAACGTAATTCCTCATATCGGA

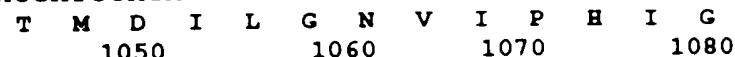
GGTGAAGAAGAAAAAGCGGAGATAGAACCTCTTAAATGTT \begin{tabular}{llllllllllllll}
\hline & $E$ & $E$ & $E$ & $K$ & $A$ & $E$ & $I$ & $E$ & $P$ & $L$ & $K$ & $C$
\end{tabular} $1090 \quad 1100 \quad 1110 \quad 1120$ TGGGTAAAGTCGAGAATGGAAAAAT T T TACACGCCGATT T $\begin{array}{llllllllllllll}I & G & K & V & E & N & G & \mathbb{K} & I & L & \text { I } & A & D & F\end{array}$ $1130 \quad 1140 \quad 1150 \quad 1160$ TTCAATTTCCGCACATTGTAACCGTGTTCCCGTTTTTGAC

$\begin{array}{lllllllllllll}S & I & S & A & \text { H } & C & N & R & V & P & V & F & D\end{array}$ $1170 \quad 1180 \quad 1190 \quad 1200$ GGTCATACGGTTTGTGTTTCCGTTAAGTTTAAAAAGAAAC

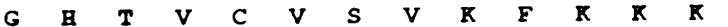
$1210 \quad 1220 \quad 1230 \quad 1240$ CTTCTAGGGAAGAAATTATTTCCTCTTGGAAAGATTTTTC

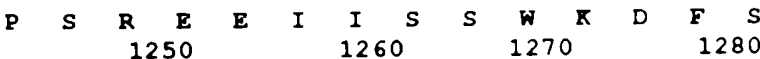
CGGAGAACCACAGACGTTAGGTCTTCCTTTAGCACCAAAT

$\begin{array}{lllllllllllll}G & \mathbf{E} & \mathbf{P} & \mathbf{Q} & \mathbf{T} & \mathbf{L} & \mathbf{G} & \mathbf{L} & \mathbf{P} & \mathrm{L} & \text { A } & \text { P } & \mathbf{N} \\ & 1290 & & & 1300 & & 1310 & & & 1320\end{array}$ CCGGTTATCCTTTTTAGAGAAGAAGAAGATAGGCCGCAAC $\begin{array}{lllllllllllll}\mathbf{P} & \mathbf{V} & \mathbf{I} & \mathbf{L} & \mathbf{F} & \mathbf{R} & \mathbf{E} & \mathbf{E} & \mathbf{E} & \mathbf{D} & \mathbf{R} & \mathbf{P} & \mathbf{Q}\end{array}$ $\begin{array}{llll}1330 & 1340 & 1350 & 1360\end{array}$ CTAGGTTAGATTTAGATACCGGCAAAGGATGACTACGGT $\begin{array}{llllllllllllll}P & R & \text { L } & D & \text { L } & D & T & G & K & G & M & T & T & \text { V }\end{array}$ $1370 \quad 1380 \quad 1390 \quad 1400$ GATAGGTCGTCTCAGGCCAGATCCTATTTTAGATTGGAAA $\begin{array}{lllllllllllll}I & G & R & L & R & P & D & P & I & L & D & W & K\end{array}$

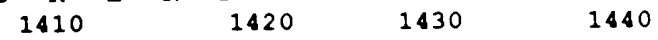
TATGTCGTTTTAAGCCATAATACAATTCGAGGAGCGGCGG

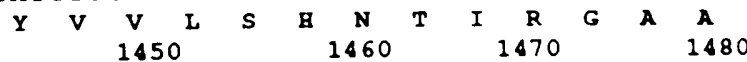
GTGCTGCATTAT TAAAT GCAGAACT TCTT TACAAAAAAAA

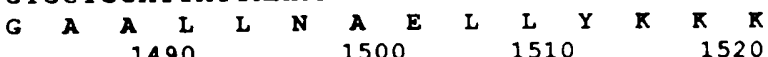
AT TCTTGgataAtaATGTCATTCCATGCTGgaACTCAa $F \quad L \quad G$

$\begin{array}{lllll}F \quad L \quad 15 & 1540 & 1550 & 1560\end{array}$

TCCCCTGAACTCAAAGTGGCGGATTACAATACCGCACTAC $1570 \quad 1580 \quad 1590 \quad 1600$

AATTGACTCAGTCGCTTGAGGCCAGAAATGACTTTCAATA $1610 \quad 1620 \quad 1630 \quad 1640$

TAAGAAAATTCATAAGCTCTTACT TATCATCTCTGATTGG $1650 \quad 1660 \quad 1670 \quad 1680$ ACAGATAAGTTCGTAGCAAATAAAGTGCTTCCTAATGTGG $1690 \quad 1700 \quad 1720 \quad 1730$

ATCAATTATCCAAGGAATTGGGTCTTGAGAAAGACAAGAC $1740 \quad 1750 \quad 1760 \quad 1770$

GTTGCAAT T TCTGAAAGAACTTTGTACAAAATACAACCCC $1780 \quad 1790 \quad 1800 \quad 1810$

CCGATCATTAAAAAAATCTGCATGGTGGATTTGAATCCTA $1820 \quad 1830$

ACGGGGATATATCTGACGGTACGATCG

Fig. 2. Nucleotide sequence of the $L$. interrogans asd gene and its flanking regions. The one-letter abbreviations for the deduced amino acid sequence of ORF1 and asd (starting at nucleotide 444) are shown below the DNA sequence.

homology with Strep. mutans aspartate $\beta$-semialdehyde dehydrogenase (Cardineau \& Curtiss, 1987). The asd gene has also been sequenced from E. coli (Haziza et al.,
1982), Sacch. cerevisiae (Thomas \& Surdin-Kerjan, 1989) and C. glutamicum (Kalinowski et al., 1990). Optimal alignment of the five deduced polypeptide 


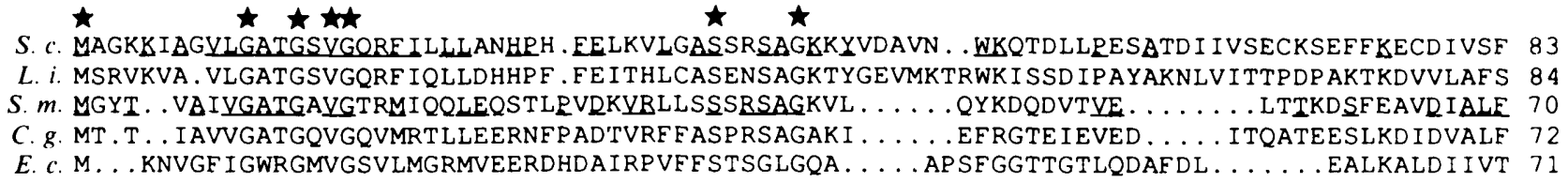

S. c. GLDADYAGA IEKEFMEAGIAI . .VSNAKNYRREQDVPLIVPVVNPEHLD IVAQKLDTAKAQGKPRPGF IICISNCSTAGLVAP ¿

L. $i$. GLDSNVAGEVEKNYANAG I H I . ISNSKNHRMDP TVP ILSAEVNSSHLEV . . . LTSQKTKGK . . . . I ITNSNCT IMGVT ISLKP 159

S. $m$. SAGGSVSAKF APYAVKAGAVY . VDNYSHFBQNPDVPLVYPEVNAYAMDAH. NG . . . . . . . ILACRNCSTIQMMVALER 139

c. g. SAGGTASKQYAPLFAAAGATV. VDNSSAWRKDDEVPLIVSEVNPSDKDSLVKG . . . . . . . I IANPNCTTMAAMPVLKP 142

E. c. CQGGDYTNE I YPKLRESGWQGYWIDAASSLRMKDDA I I I . . LDPVNQDVITDGLNNGI . . . . . RTFV. . GGNCTVSLMLMSLGG 146

tht $\star \star$

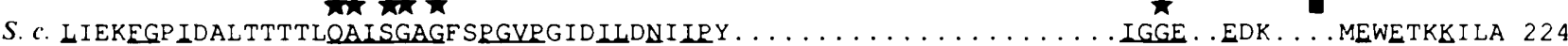

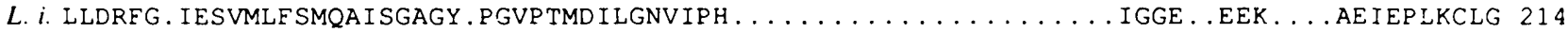

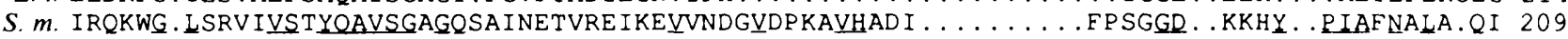

C. $g$. LHDAAG. LVKLHVSSYQAVSGSGLAGVETLAKQVAAVGDHNVE ..FVHDGQ . . . . . . AADAGD . VGPYVSPIAYNVLPFAG 213

E. c. LFAN. DLVDWVSVATYQAASGGGARHMRELLTQMGHL YGHVADELATPSSAI LD I ERKVTTLTRSGE LPVDNFGVPLAGSL I PWID 231

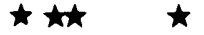

S. c. PLAEDKTHV . . . . . . . . KLLTPEE. IKVSAQCNRVAVSDGHTECISLREKNR. PAP SVEQVKTCLKEYVCDAYKLGCHSAPK 296

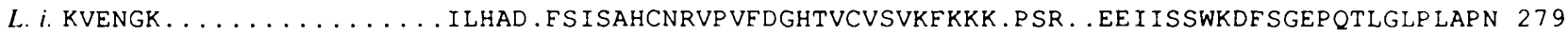

$S . m$. DVFTDNDYTY . EEMKMTNETKK IMEEEE. LPVSAHCVRVPILFSHSEAVY IETKD . . VAP IEEVKAAIAAFPGAV . . . . . 280

C. $g$. NLVDDGTFETDEEQKLRNESRK I LGLPD. LKVSGTCVRVPVFTGHT LT I HAEFDK . . AITVDQAQE I LGAASGVK . . . . . 285

E. $c$. KQLDNGQSR. . EEWKGQAETNK ILNTSSVIPVDGLCVRVGALRCHSQAFTIKLKKDVSIPTVEEL . . . . . . . . LAAHNPWA 302

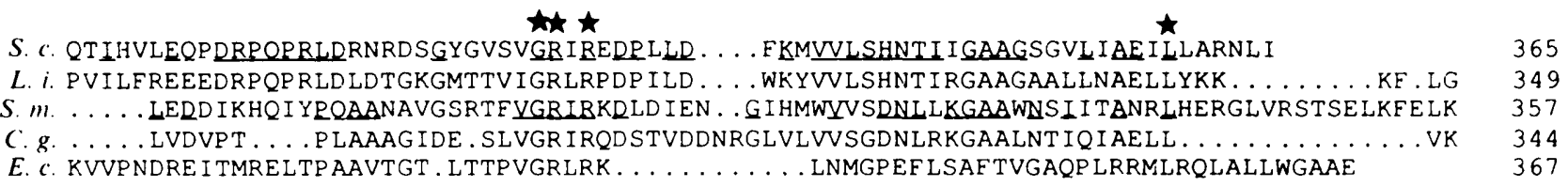

Fig. 3. Alignment of the deduced amino acid sequences of the $L$. interrogans ( $L . i$. ) asd gene product and other published aspartate semialdehyde sequences from Strep. mutans $(S$. m.), Sacch. cerevisiae $(S . c$.), C. glutamicum $(C . g$.) and E. coli (E. c.). Gaps have been introduced to maximize alignments with respect to identical and functionally similar amino acids. The positions of identical residues among all sequences are designated with stars. Identity among amino acid residues of the aspartate semialdehyde of $L$. interrogans and Sacch. cerevisiae on the one hand and of Strep. mutans and C. glutamicum on the other is represented by underlining in the upper sequence of the respective pair. The cysteine of the active site of the E. coli aspartate semialdehyde dehydrogenase (Biellmann $e t$ al., 1980; Haziza et al., 1982) is indicated by an arrow.

sequences was obtained by the CLUSTAL computer program (Higgins \& Sharp, 1988) and revealed homology between the five proteins (Fig. 3). Pairwise comparisons revealed that $L$. interrogans Asd is more closely related to Sacch. cerevisiae Asd than to the other bacterial Asd proteins. While the $L$. interrogans enzyme shows $46 \%$ identity with the enzyme from yeast, the value falls to $36 \%$ with the $C$. glutamicum enzyme, $31 \%$ with that from Strep. mutans and $25 \%$ with that from E. coli. The Strep. mutans and $C$. glutamicum enzymes share $40 \%$ of their amino acid residues but those of Sacch. cerevisiae and Strep. mutans share only $32 \%$. The polypeptide from $E$. coli is less similar to any of the other four ( 21 to $26 \%$ identity). Extensive homology between the whole Asd sequences from the five organisms provided unequivocal evidence that the genes derive from a common ancestor.

There are five regions of close similarity between the five proteins: residues 10-16, 147-148, 175-181, 232-235 and 308-311 (numbering refers to the $L$. interrogans protein, see Fig. 3). The choice of the methionine initiator codon for $L$. interrogans asd at position 444 (Fig. 2) seems reasonable given the similarity at the $\mathrm{N}$-terminus of the five sequences and is in agreement with the determined $\mathrm{N}$-terminus of the $E$. coli aspartate semialdehyde dehydrogenase (Haziza et al., 1982). No likely candidate ribosome-binding site (Shine \& Dalgarno, 1974) complementary to the $3^{\prime}$ end of the $L$. interrogans 16S rRNA (Fukunaga et al., 1990) could be found by analysis of the DNA sequence immediately upstream from the predicted ATG translational start codon of $a$ asd. The $L$. interrogans asd gene encodes a polypeptide of 349 amino acid residues with an $M_{\mathrm{r}}$ of 38007 , in good agreement with the estimated sizes of the other four Asd polypeptides.

The high degree of conservation of the amino acid sequences observed for several segments of these proteins probably reflects structural and functional requirements. In particular, the active site for the $E$. coli 
aspartate $\beta$-semialdehyde dehydrogenase has been identified as the amino acid sequence Phe-Val-Gly-Gly-AsnCys-Thr-Val-Ser (Biellmann et al., 1980; Haziza et al., 1982) between amino acid residues 130 and 138. The predicted amino acid sequence for the $L$. interrogans aspartate $\beta$-semialdehyde dehydrogenase contains a cysteine residue in the same position, with the Asn-Cys sequence that is present in the other four enzymes (see arrow in Fig. 3).

\section{Expression of the asd gene}

The asd gene of $L$. interrogans is expressed at a low level in whole $E$. coli cells since growth is possible in LB medium without DAP but enhanced when DAP is added. Confirmation of this low activity was given by the assay of aspartate semialdehyde dehydrogenase from crude extracts of $E$. coli cells (asd $:: \mathrm{Km})$ carrying the recombinant plasmid pAILl. The resulting activity was not enhanced compared to the activity from prototrophic E. coli cells expressing a single chromosomal copy of asd (data not shown). Similarly low expression is observed for the $L$. interrogans pro $A$, proB and leuB genes previously cloned, which are not expressed detectably in minicells (Richaud et al., 1990). The low expression of $L$. interrogans genes in $E$. coli could be due to translation problems (absence of canonical ribosome-binding site) and different codon usage.

The synthesis of Asd in E. coli is independently repressed by methionine, threonine and lysine, although derepression is greatest upon lysine limitation (Haziza et al., 1982). Attenuator-like regions have been found upstream of the asd gene of Strep. mutans (Cardineau \& Curtiss, 1987). The corresponding gene (HOM2) from Sacch. cerevisiae is regulated by the general control of amino acid synthesis. Upon initial inspection, the sequence upstream of the $L$. interrogans asd structural gene does not resemble the reported arrangement of attenuation signals in amino acid biosynthetic operons in Gram-negative bacteria (Kolter \& Yanofsky, 1982). This is consistent with the fact that no such signals have been found for the biosynthetic genes from L. biflexa that have been sequenced (Yelton \& Peng, 1989).

We thank B. E. Davidson, I. Old, C. Parsot and J. C. Patte for critical discussions; B. Cami and F. Borne for help in computer analysis; and D. O'Callaghan, J. Pittard and B. Bachmann for bacterial strains. This work was supported by grants from INSERM (883013), CNRS UA 1129 and the Pasteur Institute.

\section{References}

Biellmann, J. F., Eid, P., Hirth, C. \& Jörnvall, H. (1980). Aspartate semialdehyde dehydrogenase from E. coli. European Journal of Biochemistry 104, 59-64.
Brahamsha, B. \& Greenberg, E. P. (1987). Complementation of a trpE deletion in Escherichia coli by Spirochaeta aurantia DNA encoding anthranilate synthetase component I activity. Journal of Bacteriology 169, 3764-3769.

Cardineau, G. A. \& Curtiss, R., III (1987). Nucleotide sequence of the asd gene of Streptococcus mutans: identification of the promoter region and evidence for an attenuator-like sequence preceding the structural gene. Journal of Biological Chemistry 262, 3344-3353.

Charon, N. W., Johnson, R. C. \& Peterson, D. (1974). Amino acid biosynthesis in the spirochete Leptospira: evidence for a novel pathway of isoleucine synthesis. Journal of Bacteriology 117, 203-211.

Fukunaga, M., Horie, I., Okuzako, N. \& Mifuchi, I. (1990). Nucleotide sequence of a 16S rRNA gene for Leptospira interrogans serovar canicola strain Moulton. Nucleic Acids Research 18, 366.

Haziza, C., Stragier, P. \& PatTe, J. C. (1982). Nucleotide sequence of the asd gene of Escherichia coli: absence of a typical attenuation signal. EMBO Journal 1, 379-384.

Higgins, D. G. \& Sharp, P. M. (1988). Clustal: a package for performing multiple sequence alignment on a microcomputer. Gene 73, 237-244.

Johnson, R. C. (1977). The spirochetes. Annual Review of Microbiology 31, 89-106.

Kalinowski, J., Bachmann, B., Thierbach, G. \& Pühler, A. (1990). Aspartokinase genes $l y s C \alpha$ and $l y s C \beta$ overlap and are adjacent to the aspartate $\beta$-semialdehyde dehydrogenase gene asd in Corynebacterium glutamicum. Molecular and General Genetics 224, 317-324.

KolTER, R. \& YANOFSKY, C. (1982). Attenuation in amino acid biosynthetic operons. Annual Review of Genetics 16, 113-134.

Martinez, E., Bartolomé, B. \& De la Cruz, F. (1988). pACYC184derived cloning vectors containing the multiple cloning site and lac $Z \alpha$ reporter gene of $\mathrm{pUC} 8 / 9$ and $\mathrm{pUC18} / 19$ plasmids. Gene 68, $159-162$.

Novel, G. \& Novel, M. (1973). Mutants d'Escherichia coli K12 affectés pour leur croissance sur methyl- $\beta$-glucuronide: localisation du gène de structure de la $\beta$-D-glucuronidase (nidA). Molecular and General Genetics 120, 319-335.

PARsot, C. (1986). Evolution of biosynthetic pathways: a common ancestor for threonine synthase, threonine dehydratase and D-serine dehydratase. EMBO Journal 5, 3013-3019.

PittaRd, J. \& Wallace, B. J. (1966). Distribution and function of genes concerned with aromatic biosynthesis in Escherichia coli. Journal of Bacteriology 91, 1494-1508.

Richaud, C., Margarita, D., Baranton, G. \& Saint Girons, I. (1990). Cloning of genes required for amino acid biosynthesis from Leptospira interrogans serovar icterohaemorrhagiae. Journal of General Microbiology 136, 651-656.

Richaud, C., Richaud, F., Martin, C., Haziza, C. \& Patte, J. C. (1984). Regulation of expression and nucleotide sequence of the Escherichia coli dapD gene. Journal of Biological Chemistry 259, 14824-14828.

Sambrook, J., Fritsch, E. F. \& Maniatis, T. (1989). Molecular Cloning: a Laboratory Manual, 2nd edn. Cold Spring Harbor, NY: Cold Spring Harbor Laboratory.

SANGER, F., Nicklen, S. \& Coulson, A. R. (1977). DNA sequencing with chain-terminating inhibitors. Proceedings of the National Academy of Sciences of the United States of America 74, 5463-5467.

Segers, R. A. M., Van der Drift, A., De Nius, A., Corcione, P., Van DER ZEIJST, B. A. \& GAAstra, W. (1990). Molecular analysis of a sphingomyelinase $\mathrm{C}$ gene from Leptospira interrogans serovar hardjo. Infection and Immunity 58, 2177-2185.

Shapira, S. K., Chou, J., Richaud, F. V. \& Casadaban, M. J. (1983). New versatile plasmid vectors for expression of hybrid protein coded by a cloned gene fused to lac $Z$ gene sequences encoding an enzymatically active carboxy-terminal portion of $\beta$-galactosidase. Gene 25, 71-82.

Shine, J. \& Dalgarno, L. (1974). The 3' terminal sequence of Escherichia coli $16 \mathrm{~S}$ ribosomal RNA: complementarity to nonsense triplets and ribosome binding sites. Proceedings of the National Academy of Sciences of the United States of America 71, 1342-1346.

SOUTHERN, E. (1975). Detection of specific sequences among DNA fragments separated by gel electrophoresis. Journal of Molecular Biology 98, 503-517. 
ThOMAS, D. \& SURDIN-KERJAN, Y. (1989). Structure of the HOM 2 gene of Saccharomyces cerevisiae and regulation of its expression. Molecular and General Genetics 217, 149-154.

Winans, S. C., Elledge, S. J., Krueger, J. H. \& Walker, G. C (1985). Site-directed insertion and deletion mutagenesis with cloned fragments in Escherichia coli. Journal of Bacteriology 161, 12191221.

YeLTON, D. B. \& CHARON, N. W. (1984). Cloning of a gene required for tryptophan biosynthesis from Leptospira biflexa serovar patoc into Escherichia coli. Gene 28, 147-152.
Yelton, D. B. \& COHEN, R. A. (1986). Analysis of cloned DNA from Leptospira biflexa serovar patoc which complements a deletion of the Leptospira trpE gene. Journal of Bacteriology 165, 41-46.

Yelton, D. B. \& PENG, S. L. (1989). Identification and nucleotide sequence of the Leptospira biflexa serovar patoc trpE and $\operatorname{trp} G$ genes. Journal of Bacteriology 171, 2083-2089.

ZUERNER, R. L. \& CHARON, N. W. (1988). Nucleotide sequence analysis of a gene cloned from Leptospira biflexa serovar patoc which complements an argE defect in Escherichia coli. Journal of Bacteriology 170, 4548-4554. 